04

\title{
Методика измерений двойным зондом в электродном разряде с изменяющимися параметрами
}

\author{
(C) А.С. Барышников, И.В. Басаргин, Н.О. Безверхний, С.В. Бобашев, Н.А. Монахов, П.А. Попов, \\ В.А. Сахаров, М.В. Чистякова
}

Физико-технический институт им. А.Ф. Иофрфе РАН, 194021 Санкт-Петербург, Россия

e-mail: nm1988@mail.ru

Поступило в Редакцию 23 июля 2019 г.

В окончательной редакции 23 июля 2019 г.

Принято к публикации 28 августа 2019 г.

\begin{abstract}
Предложена методика корректировки сигнала двойного зонда при измерениях в плазме изменяющегося во времени электродного разряда. Методика проверена в экспериментах по взаимодействию ударной волны с поперечным тлеющим разрядом в режиме стационарного горения и распадающейся плазмы.
\end{abstract}

Ключевые слова: двойной зонд, ударная волна, горение, тлеющий разряд.

DOI: $10.21883 /$ JTF.2020.02.48810.275-19

\section{Введение}

Метод электрических зондов для диагностики плазмы используют для изучения стационарных и импульсных тлеющих разрядов, плазмы послесвечения, ионизованных газов в верхних слоях атмосферы, плазменных сгустков и струй, продуктов сгорания, плазмы МГД-систем, термоэмиссионных преобразователей, источников света, газоразрядных приборов и т.п. [1-8]. В экспериментальных исследованиях газоразрядной плазмы двойной электрический зонд находит широкое применение [9-14] в первую очередь благодаря малому возмущению исследуемой среды, вносимому зондом, поскольку ток зонда ограничен ионным током насыщения. Другое преимущество двойного зонда состоит в том, что зонд, находясь под плавающим потенциалом, позволяет проводить измерения в безэлектродной плазме или в неограниченном пространстве [15-17]. Однако в плазме электродного разряда при изменении его параметров, например из-за прохождения ударной волны, результаты измерений могут искажаться. В частности, при изменении потенциала пространства возникает ток, связанный с перезарядом емкости зонда через заземленный электрод разрядного промежутка. Если ток перезаряда протекает через элементы измерительной цепи, то результат измерения будет содержать ошибку. Авторами предложен метод коррекции результатов измерений, выполненных двойным зондом в плазме тлеющего разряда, параметры которого изменяются во времени.

Зонд, помещенный в плазму разряда, накапливает заряд, величина которого определяется электрической емкостью зонда и потенциалом окружающего пространства. На рис. 1 показаны участок вольт-амперной характеристики (BAX), соответствующий режиму насыщения ионного тока (верхняя часть), и электрическая схема двойного зонда (нижняя часть). Между электродами зон- да 1 и 2 включен источник напряжения $E$, под действием которого возникает ионный ток $I_{i}$. Величина ионного тока определяется концентрацией ионов в окрестности отрицательного электрода 1 [17].

Емкости электродов зонда, обозначенные на схеме соответственно $C_{1}$ и $C_{2}$, в условиях стационарного горения разряда заряжены до некоторого постоянного напряжения относительно заземленного электрода разрядного промежутка. Изменение потенциала пространства вызывает изменение заряда на них. Ток перезаряда $I_{C}$ этих емкостей будет проходить преимущественно через положительный электрод 2. Это обусловлено различием потенциала электродов зонда относительно плазмы, что видно на ВАХ зонда (рис. 1): потенциал положительного электрода 2 близок к потенциалу плазмы в отличие от электрода 1.

Эти рассуждения были проверены путем экспериментального измерения тока в цепи отрицательного и положительного электродов при изменении их потенциала относительно постоянного потенциала плазмы в разряде. Опыт показал, что практически весь ток перезаряда емкости зонда проходит через положительный электрод. Следовательно, и при изменении потенциала плазмы перезаряд будет осуществляться также через положительный электрод. На рис. 1 направления токов разряда емкостей показаны штриховыми линиями. Поскольку только цепь перезаряда емкости $C_{1}$ включает измеритель тока $A$, результат измерения является суммой ионного тока $I_{i}$ и тока перезаряда $I_{C}$ емкости отрицательного электрода зонда.

Величина ионного тока зависит от параметров плазмы около отрицательного электрода 1 , а ток перезаряда определяется параметрами плазмы в окрестности положительного электрода 2. Это обстоятельство позволяет изменить ионный ток за счет изменения площади отрицательного электрода при сохранении величины 

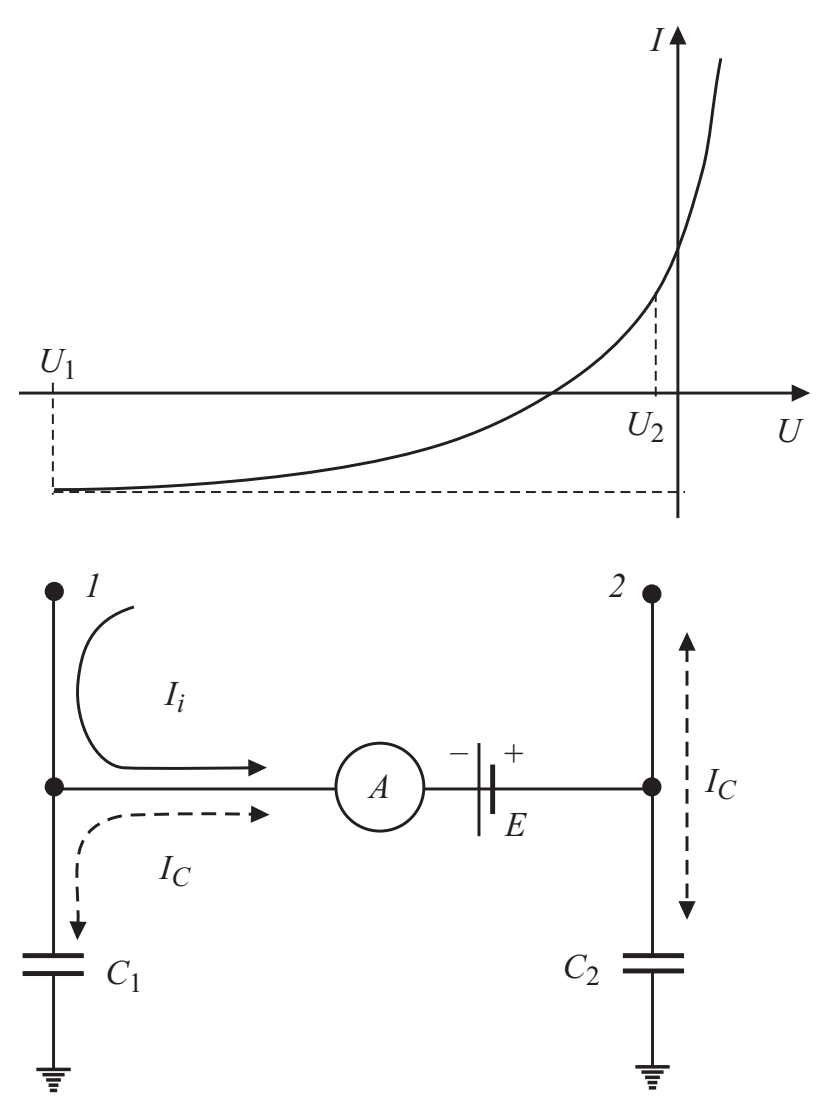

Рис. 1. Схема измерения.

тока перезаряда. Имея два измеренных значения тока двойного зон $I_{1}$ и $I_{2}$, соответствующих разной площади поверхности отрицательного электрода, можно составить систему двух линейных уравнений:

$$
\left\{\begin{array}{l}
I_{i}+I_{C}=I_{1} \\
\alpha I_{i}+I_{C}=I_{2}
\end{array},\right.
$$

где $I_{i}, I_{C}-$ искомые ионный ток и ток перезаряда, $0<\alpha<1$ - относительное изменение площади поверхности отрицательного электрода зонда. Величина $\alpha$ постоянна и может быть определена, как отношение токов, измеренных при разной площади поверхности отрицательного электрода в стационарно горящем разряде. Получить два измерения, соответствующих разной площади поверхности отрицательного электрода, можно двумя способами. Первый заключается в проведении двух опытов, измерения в которых проводятся одним зондом с изменяемой длиной отрицательного электрода, второй - в использовании в одном опыте двух двойных зондов с разной длиной отрицательного электрода. Для достижения наилучшей точности результата измерений в условиях нашей экспериментальной установки предпочтительным оказался второй вариант.

Решение системы (1) имеет вид

$$
\left\{\begin{array}{l}
I_{i}=\frac{I_{1}+I_{2}}{1-\alpha} \\
I_{C}=\frac{I_{2}-\alpha I_{1}}{1-\alpha}
\end{array},\right.
$$

из чего следует, что, зная значение коэффициента $\alpha$, можно получить величины $I_{i}$ и $I_{C}$ из измеряемых значений тока двойного зон $I_{1}$ и $I_{2}$.

Для того чтобы возмущение плазмы, вносимое зондом, оставалось малым, необходимо, чтобы ток перезаряда $I_{C}$ был одного порядка с ионным током $I_{i}$. Это ограничение можно также считать условием, при котором применим предлагаемый метод коррекции результатов измерений.

\section{Эксперимент}

Проверка предлагаемой методики проводилась на установке для исследования взаимодействия ударной волны с плазмой тлеющего разряда [17-22]. Установка представляла собой цилиндрическую камеру диаметром $300 \mathrm{~mm}$ и высотой $400 \mathrm{~mm}$, соединенную с ударной трубой. В рабочей камере между вертикально расположенными электродами создавался постоянно горящий тлеющий разряд в воздушной атмосфере при давлении $\sim 4 \mathrm{kPa}$. Электроды подключены к источнику питания, который обеспечивает ток разряда $\sim 1.1 \mathrm{~A}$ при напряжении на электродах $\sim 680 \mathrm{~V}$. Разряд реализуется в свободном пространстве вдали от стенок камеры, диаметр сечения видимой части разряда составляет

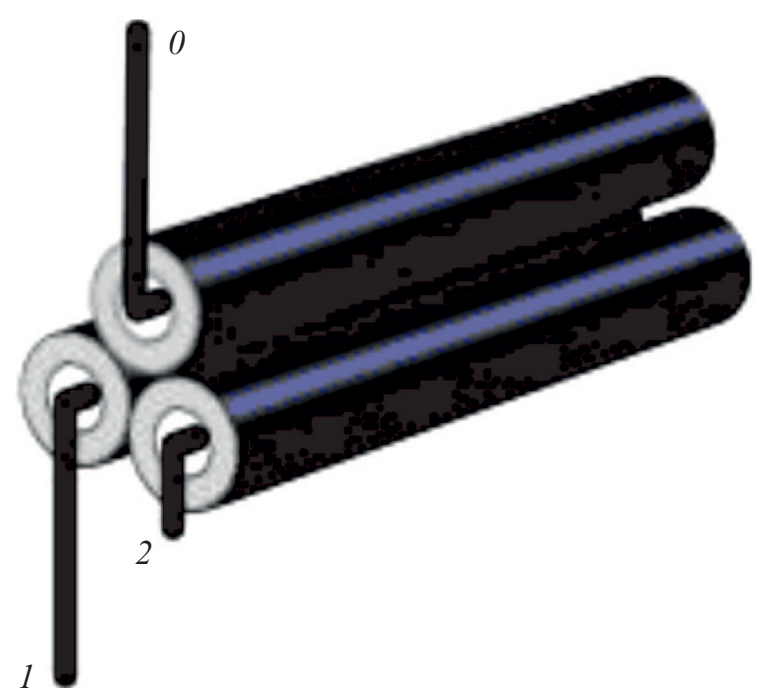

Рис. 2. Компоновка трехэлектродного зонда: $O-$ общий положительный, 1 и 2 - отрицательные электроды.

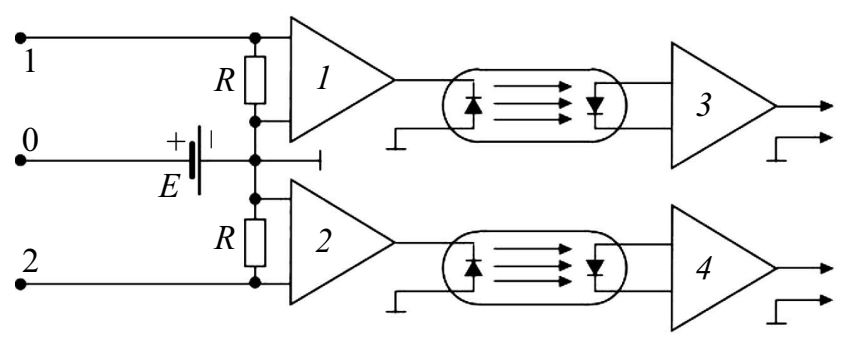

Рис. 3. Схема регистрации тока трехэлектродного зонда. 


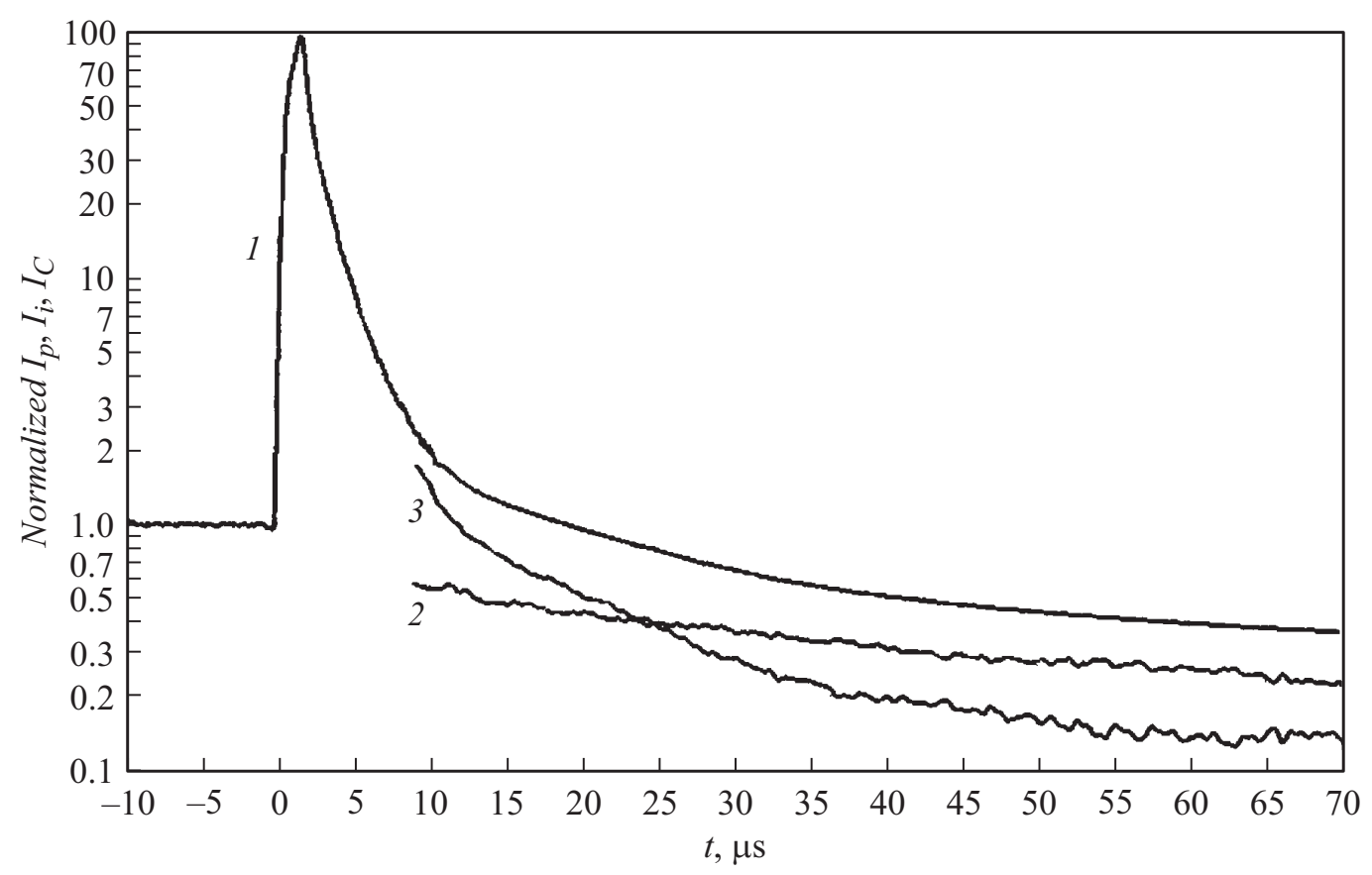

Рис. 4. Изменение тока зонда при выключении тока разряда. $1-$ ток зонда $\left(I_{p}\right), 2-$ ионный ток $\left(I_{i}\right), 3-$ ток разряда емкости зонда $\left(I_{C}\right)$.

менее $\sim 1 / 3$ диаметра рабочей камеры. Ударная волна генерировалась с помощью электроразрядной ударной трубы, расположенной перпендикулярно оси разряда на расстоянии $150 \mathrm{~mm}$ от нее. Электрический зонд располагался соосно с ударной трубой на расстоянии $20 \mathrm{~mm}$ от оси разряда с противоположной стороны.

Для измерений использовался зонд с тремя электродами, один из которых являлся общим для обоих зондов. Каждый электрод зонда был расположен внутри кварцевого капилляра и выступал за его край. Выступ короткого электрода составлял $3 \mathrm{~mm}$, двух длинных $10 \mathrm{~mm}$. Поиск оптимального взаимного расположения электродов зонда, по-видимому, также следует связать с особенностями конкретной задачи. В нашем случае наиболее удачным оказалось расположение, показанное на рис. 2. Общий электрод 0 подключался к положительному полюсу источника напряжения, а электроды 1 и $2-$ к отрицательному. Основной недостаток зонда с тремя электродами заключается в том, что электроды 1 и 2 (рис. 2) расположены в различных точках пространства. Наличие градиента концентрации заряженных частиц вдоль линии соединения этих электродов может быть источником погрешности при обработке результатов измерений.

На рис. 3 показана электрическая схема регистрации токов зонда с тремя электродами. К положительному полюсу источника напряжения $E$ подключен общий электрод зонда $O$ (рис. 2). Общий источник питания в измерительной цепи обеспечивает равные напряжения на отрицательных электродах 1 и 2 . Сигнал с нагрузки $R$ в цепи каждого зонда подается на соответствующий операционный усилитель 1 и 2. Поскольку зонды в плазме разряда находятся под высоким напряжением относительно земли, необходима гальваническая развязка между зондами и регистрирующей аппаратурой. С этой целью использованы оптроны с последующим усилением сигнала операционными усилителями 3 и 4, выходные сигналы которых регистрируются на осциллографе.

Один из этапов проверки состоял в проведении измерений в условиях прогнозируемого изменения ионного тока. В качестве такого опыта использовался режим быстрого отключения тока разряда. Этот прием применяется при создании распадающейся плазмы. Отключение тока разряда осуществлялось в течение не более $2 \mu \mathrm{s}$. За это время происходит обнуление напряжения на аноде разрядного промежутка, а потенциал пространства в окрестности зонда уменьшается от $400 \mathrm{~V}$ до нуля.

Другой этап проверки методики состоял в сравнении реакции зонда на движение ударной волны в плазме тлеющего разряда и распадающейся плазме. Взаимодействие ударной волны с распадающейся плазмой происходит через $70 \mu$ s после отключения тока разряда, когда, с одной стороны, нет влияния переходного процесса, с другой стороны, величина ионного тока соизмерима с его начальным значением.

\section{Результаты}

На рис. 4 показаны измеренный сигнал тока одного из зондов, возникающий при отключении тока разряда, и 


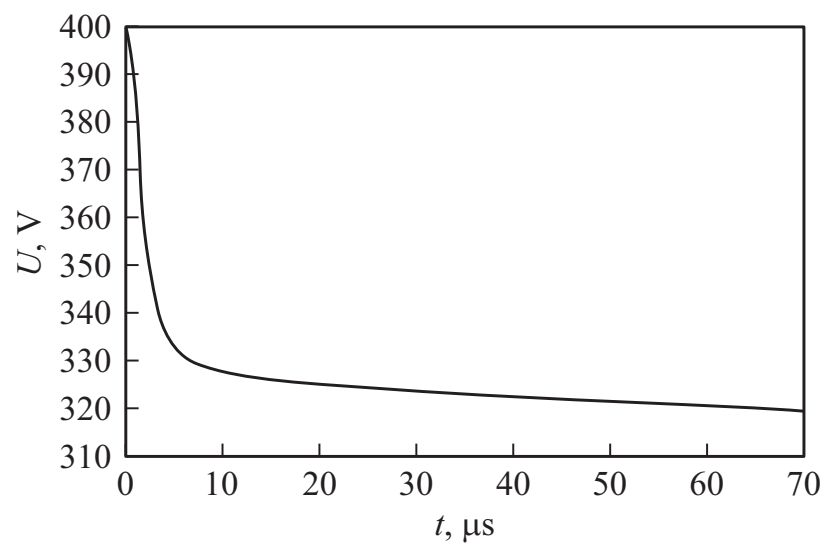

Рис. 5. Изменение напряжения на зонде.

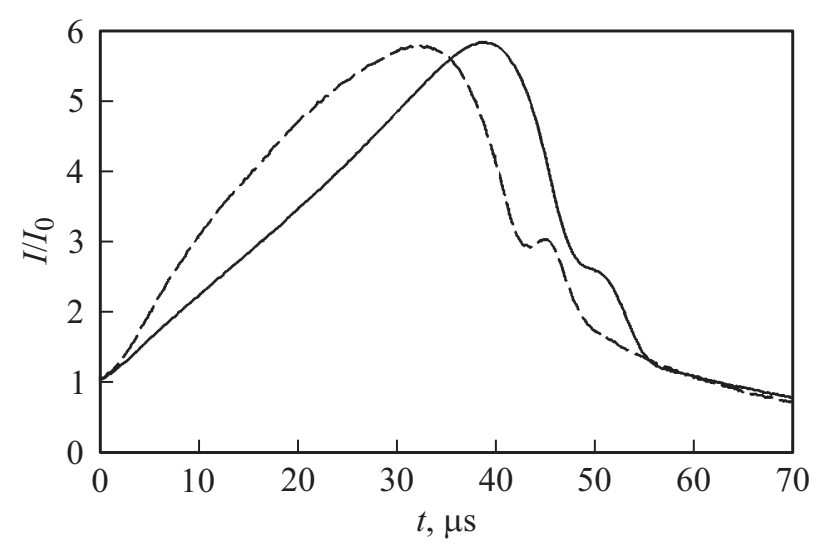

Рис. 6. Изменение ионного тока за фронтом ударной волны в тлеющем разряде (сплошная линия) и распадающейся плазме (штриховая линия).

результат коррекции. Значения тока зонда нормированы на его величину в режиме постоянного горения, т.е. на начальную величину ионного тока. Отсчет времени ведется от момента снятия напряжения на разрядном промежутке. Отрицательные значения времени соответствуют режиму постоянного горения тлеющего разряда.

На рис. 4 видно, что измеренный зондовый ток $I_{p}$ (линия 1) резко возрастает, достигая максимума примерно через $2 \mu$ s от начала процесса. Ток разряда в этом случае на два порядка больше ионного тока. Результат коррекции - ионный ток $I_{i}$ (линия 2) и ток разряда емкости зонда $I_{C}$ (линия 3 ) в интервале от 0 до $10 \mu \mathrm{s}$ определить не удается по причине того, что величина тока разряда существенно превышает величину ионного тока. После $10 \mu \mathrm{s}$, когда величины составляющих тока зонда становятся одного порядка, ионный ток и ток разряда монотонно уменьшаются: первый вследствие рекомбинации плазмы, второй - разряда емкости электрода зонда.

Интеграл по времени от тока разряда емкости зонда отражает изменение заряда на зонде. При известной величине емкости зонда можно построить изменение напряжения на нем. На рис. 5 показана зависимость напряжения на зонде, начальное значение которого в рассматриваемом примере составляет $400 \mathrm{~V}$. Видно, что в течение $10 \mu \mathrm{s}$ напряжение уменьшается существенно, а далее - сравнительно медленно. Емкость зонда остается заряженной длительное время после выключения тока разряда. Поскольку разряд емкости зонда через положительный электрод является преимущественной, но не единственной электрической цепью, полученное изменение напряжения на зонде (рис. 5) представляет собой минимальную оценку.

На рис. 6 показано изменение ионного тока за фронтом ударной волны в плазме разряда (сплошная линия) и распадающейся плазме (штриховая линия). Значения ионного тока отнесены к его величине перед ударной волной, а время в этом случае отсчитывается от момента прихода волны к зонду. На рисунке видно, что величина газодинамического сжатия плазмы, в том числе и заряженных частиц, в обоих случаях совпадает. При этом движение ударной волны в распадающейся плазме сопровождается уменьшением потенциала пространства, а в плазме тлеющего разряда - его увеличением.

Следует заметить, что аналогичное сопоставление измеренных сигналов зонда с равной длиной электродов обнаруживает несовпадение величины максимумов более чем на $10 \%$ [15]. Это расхождение многократно увеличивается при сравнении соответствующих сигналов зонда с разной длиной электродов.

\section{Заключение}

В работе предложена методика корректировки сигнала двойного зонда при измерениях в плазме электродного разряда, параметры которого изменяются во времени. Методика была проверена в экспериментах по взаимодействию ударной волны с поперечным тлеющим разрядом. Проведенное тестирование методики корректировки подтверждает наши представления о физических процессах, протекающих при нестационарных измерениях параметров плазмы тлеющего разряда с помощью двойных зондов. Экспериментальные результаты показывают, что применение предложенной методики корректировки сигнала двойного зонда позволяет получить более точную информацию об объекте исследования. В ходе измерений было показано, что газодинамическое сжатие плазмы одинаково для случаев прохождения ударной волны по плазме тлеющего разряда и по распадающейся плазме.

\section{Конфликт интересов}

Авторы заявляют, что у них нет конфликта интересов. 


\section{Список литературы}

[1] Adams S.F., Miles J.A., Demidov V.I. // Phys. Plasmas. 2017. Vol. 24. P. 053508.

[2] Megalingam M., Prakash N. Hari, Solomon I. et al. // Phys. Plasmas. 2017. Vol. 24. P. 042304.

[3] Popov T., Tsaneva V.N., Stelmashenko N.A. et al. // Plasma Sources Sci. Technol. 2005. Vol. 14. N 1. P. 184.

[4] Naz M.Y., Ghaffar A., Naseer S., Zakaullah M. // Prog. Electromagn. Res. 2011. Vol. 114. P. 113-128.

[5] Eckman R.F., Byrne L., Gatsonis N.A., Pencil E.J. // J. Propul. Power. 2001. Vol. 17. N 4. P. 762-771.

[6] Tanışlı M., Şahin N., Demir S. // Can. J. Phys. 2018. Vol. 96. N 5. P. 494-500.

[7] Kato K., Iizuka S. // JAMP. 2016. Vol. 4. N 9. P. 1811-1836.

[8] Godyak V.A., Alexandrovich B.M. // J. Appl. Phys. 2015. Vol. 118. N 23. P. 233302.

[9] Каган Ю.М., Перель В.И. // УФН. 1963. № 81. С. 409-452.

[10] Clements R.M. // J. Vac. Sci. Technol. 1978. Vol. 15. N 2. P. $193-198$.

[11] Чан П., Тэлбот Л., Турян К. Электрические зонды в неподвижной и движущейся плазме М.: Мир, 1978.

[12] Демидов В.И., Колоколов Н.Б., Кудрявцев А.А. Зондовые методы исследования низкотемпературной плазмы. М.: Энергоатомиздат, 1996.

[13] Marek A. et al. // Contrib. Plasma Phys. 2008. Vol. 48. N 5-7. P. 491-496.

[14] Naz M.Y. et al. // Measurement. 2016. Vol. 91. P. 194-200.

[15] Альперт Я.Л., Гуревич А.В., Питаевский Л.П. Искусственные спутники в разреженной плазме. М.: Наука, 1964.

[16] Зиятдинов P.X. // Вестник Казанского технол. ун-та. 2014. T. 17. C. 245-247.

[17] Райзер Ю.П. Физика газового разряда. Долгопрудный: ИД Интеллект, 2009.

[18] Барышников А.С., Басаргин И.В., Бобашев С.В. и др. // ИФЖ. 2016. Т. 89. № 3. С. 555-558.

[19] Барышников А.С., Басаргин И.В., Бобашев С.В., Монахов Н.А., Попов П.А., Сахаров В.А., Чистякова М.В. // Письма в ЖТФ. 2017. Т. 43. Вып. 11. С. 20-25.

[20] Барышников А.С., Басаргин И.В., Безверхний Н.О., Бобашев С.В., Монахов Н.А., Попов П.А., Сахаров В.А., Чистякова М.В. // ЖТФ. 2018. Т. 88. Вып. 2. С. 180-182.

[21] Baryshnikov A.S., Basargin I.V., Bezverkhnii N.O. et al. // AIAA J. 2018. Vol. 56. N 9. P. 3782-3784.

[22] Baryshnikov A.S., Basargin I.V., Bezverhniy N.O. et al. // J. Phys.: Conf. Ser. 2018. Vol. 1112. P. 012007. 